\title{
Les incertitudes \\ du soi et la \\ question du bon \\ modèle théorique \\ en immunologie
}

et décisive au cours $d u x^{\mathrm{e}}$ siècle, non seulement au sein de la biologie, mais également dans l'ensemble de la société. Derrière les défis les

Thomas Pradeu plus pressants de l'immunologie, au premier rang desquels le sida ou le traitement des cancers, se profilent tous les enjeux médicaux d'aujourd'hui et de demain. Dans ces conditions, il peut parâ̂tre essentiel de disposer, en immunologie, de fondements théoriques solides, susceptibles d'orienter les recherches vers d'importantes et très attendues découvertes. Cependant, l'immunologie est aujourd'hui dans une situation d'interrogation sur elle-même : le modèle du soi, qui peut être considéré comme le fondement conceptuel de la discipline, est très critiqué, sans qu'aucune autre théorie n'ait réussi à cristalliser autour d'elle un consensus. Dès lors, plusieurs questions se posent: quels sont les principes et les origines du modèle du soi? Ce modèle a-t-il échoué dans son objectif d'expliquer les observations disponibles en immunologie? Une science aussi indispensable à nos sociétés peutelle se développer sans perspectives ni horizon théoriques? <

L'immunologie a offert à la médecine nombre de ses découvertes fondamentales et un langage propre, en particulier au cours des cinquante dernières années, avec la terminologie du soi et du non-soi [1]. Son influence, cependant, ne s'est pas limitée à la médecine, mais s'est étendue, à l'heure du sida, aux sociétés dans leur ensemble. Or, l'immunologie contemporaine doit faire face à un paradoxe. D'un côté, elle n'a cessé d'être, depuis le début du $x x^{e}$ siècle, l'une des sciences expérimentales les plus théoriques et même les plus philosophiques, comme l'illustrent Elie Metchnikoff, Paul Ehrlich, Niels Jerne et surtout Frank M. Burnet, qui a affirmé que l'immunité était plus une question de philosophie que de pratique scientifique [2]! Mais, d'un autre côté, elle semble ne plus disposer aujourd'hui d'une théorie admise par tous,

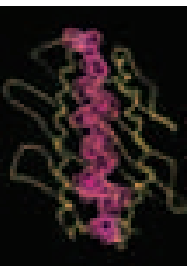

et donc être en quête d'un cadre théorique nouveau, capable de rendre compte des données disponibles et de guider les recherches médicales vers des finaliInstitut d'histoire et de philosophie des sciences et des techniques (IHPST), Université Paris 1 PanthéonSorbonne, 13, rue du Four, 75006 Paris, France.

thomas.pradeu@ens.fr tés précises. Ainsi, après une analyse historique du concept du soi, nous présenterons les défis adressés au modèle du soi et tenterons de dessiner les grandes lignes d'une réorientation théorique de l'immunologie.

\section{Une brève histoire du « soi »}

Metchnikoff, à la fin du XIXe siècle, a établi un lien fort entre l'immunité et la définition de l'identité de l'organisme [3], sans employer le terme «soi ». Metchnikoff plaçait l'inflammation, et non la défense, au cœur de l'immunité, et n'assimilait pas identité de l'organisme et défense de son intégrité [4]. Pour lui, l'organisme ne définit pas son identité par sa préservation contre toute influence étrangère, mais par un échange constant entre l'intérieur (l'organisme lui-même) et l'extérieur (l'environnement). Or, c'est précisément l'assimilation de l'immunité et de la défense de l'intégrité qui est devenue caractéristique de la notion de «soi » avec Ehrlich et Burnet.

Le principe du modèle du soi et du non-soi, formulé par Burnet [5], est qu'un organisme déclenche une réaction immunitaire contre toute entité qui lui est étrangère (non-soi), alors qu'il tolère toute entité qui lui est propre (soi). Tout ce qui déroge à ce principe est considéré comme une exception, par définition rare. Le soi est l'identité-forteresse, noyau de l'individualité que l'orga- 
nisme doit protéger contre toute menace extérieure. La domination du modèle du soi, indéniable à partir des années 50 [6], est notamment liée au fait qu'il a été conforté par plusieurs expériences : rejet/tolérance de greffes [7], démonstration de la sélection négative [8], découverte du complexe majeur d'histocompatibilité, tendant à prouver que l'immunité s'appuie sur un véritable «système de reconnaissance » du soi [9].

Mais que signifie exactement le soi en immunologie? Ce terme a de riches racines conceptuelles. Pour les comprendre, remontons aux «sources» du soi : immunologiques proprement dites, écologico-biologiques, philosophiques.

\section{Sources immunologiques}

L'origine du soi immunologique se trouve chez Ehrlich $[10,11]$, qui propose le principe de l'horror autotoxicus, selon lequel il est inconcevable qu'un organisme déclenche une réaction immunitaire contre lui-même. La thèse de Burnet prolonge celle d'Ehrlich [12], mais Burnet avait connaissance de l'existence des auto-anticorps et avait constaté que les nouveau-nés, chez certains animaux, n'ont guère de défenses immunitaires: pour lui, le problème était de comprendre comment les acteurs de l'immunité apprennent la tolérance au soi, c'est-à-dire à ne pas attaquer le soi. Burnet propose ainsi une vision négative et dynamique du soi : ce dernier n'est pas fixe, il représente l'ensemble des motifs auxquels les cellules immunitaires ont appris à ne pas réagir. Burnet propose, pour la première fois, un critère d'immunogénicité (principe explicatif permettant de dire dans quels cas il y aura une réaction immunitaire), ce qui constitue une avancée théorique décisive : l'immunologie passe de la simple description à une véritable hypothèse prédictive, susceptible d'être infirmée ou confirmée.

\section{Sources écologico-biologiques}

Burnet a été profondément influencé par l'ouvrage The Science of Life [13] dans sa conception écologique des organismes et dans le recours au terme «soi » [14]. Ce livre a marqué la biologie du début du xx siècle par l'audace de ses thèses, en raison notamment de la créativité de Julian Huxley. Pour les auteurs, l'organisme était assimilable à une communauté peuplée de pathogènes engagés dans une lutte perpétuelle. En outre, ils établissaient une analogie entre l'organisme et le soi psychologique défini, dans le prolongement de Freud et Jung, comme une réalité en mouvement continu, constituée par ses intégrations et ses rejets, analogie dont Burnet s'inspire à son tour. Cependant, Burnet ressent rapidement la nécessité de figer ce soi dynamique et ouvert, car il souhaite l'ancrer dans les déterminations génétiques [15]. Il affirme alors que l'ouverture à l'environnement (caractéristique de la vision écologique) concerne seulement l'embryon ou le nouveau-né (avant la sélection lymphocytaire), tandis que l'organisme se ferme ensuite, la défense de son intégrité passant désormais par la non-tolérance de toute substance étrangère.

\section{Sources philosophiques}

Au-delà des antécédents biologiques, écologiques et psychologiques, la source première du soi immunologique semble bien être la philosophie. Un usage substantivé du terme «soi» dans le sens d'ego, ce qu'un individu est intrinsèquement, se trouve dans l'Essai sur l'entendement humain de John Locke ([16], Livre II, chapitre 27). En philosophie, le soi désigne l'être individuel pensant, dans sa double dimension d'unicité et de maintien dans le temps. Le soi psychologique étant lui-même dérivé du soi philosophique, ce dernier apparaît bien comme l'origine la plus lointaine du soi immunitaire. En plaçant le terme soi au centre de son édifice théorique, l'immunologie emprunte l'idée de noyau intrinsèque qui le fonde en philosophie, et donc une conception substantialiste de l'être vivant. La substance d'un être est le noyau métaphysique de son individualité, qui se maintient en dépit des changements qui l'affectent. Le soi immunitaire désigne ce que je suis intrinsèquement et qui est préservé à travers le temps, le noyau d'intégrité que tout organisme défend contre les agressions extérieures [17].

\section{Imprécisions du terme « soi »}

Le terme «soi » se révèle, à l'analyse, particulièrement équivoque et imprécis. Le soi immunitaire oscille entre quatre définitions: l'organisme dans son ensemble ([5], p. 85), le génome de l'individu [15], l'ensemble des peptides présentés aux immunocytes lors de leur maturation ([18], p. 16) et, définition tautologique, ce qui ne déclenche pas de réaction immunitaire [19]. Les partisans du modèle du soi préfèrent la plupart du temps ne pas faire de choix entre ces différentes significations et conserver au terme «soi» son sens le plus élastique. Aucune science, pourtant, ne saurait se contenter d'une telle imprécision conceptuelle; elle doit trouver un équilibre entre exactitude et ouverture d'esprit à diverses interprétations possibles [20]. Dès lors, plusieurs épistémologues de l'immunologie ont considéré le soi immunitaire comme une simple métaphore, ou du moins comme un terme qu'il est impossible de considérer comme scientifique [3, 12], une sorte d'axiome destiné à donner l'illusion d'un consensus par le recours à un vocabulaire non défini.

\section{Confrontation du modèle du soi aux données expérimentales contemporaines}

Aux imprécisions conceptuelles du modèle du soi et du non-soi sont venus s'ajouter, depuis une vingtaine d'années, des doutes sur son adéquation aux données expérimentales actuelles.

\section{Autoréactivité}

Plusieurs équipes ont montré, depuis les années 90, que les lymphocytes $T$ ne survivent lors de la sélec- 
tion lymphocytaire que s'ils réagissent faiblement au soi (et non s'ils n'y réagissent pas du tout), et qu'ils ne survivent ultérieurement dans l'organisme que s'ils sont continûment stimulés par des constituants du soi [21]. En outre, la redécouverte, depuis dix ans, des cellules T régulatrices a confirmé l'importance des phénomènes d'auto-immunité : ces cellules réagissent à d'autres cellules immunitaires en régulant l'intensité de leur réponse [22]. Les réactions immunitaires au soi sont donc un phénomène nécessaire et constant dans l'organisme, et non une exception pathologique.

\section{Apports récents dans le domaine de la tolérance immunitaire}

Bien que la tolérance immunitaire, phénomène dans lequel un organisme ne rejette pas des entités pourtant étrangères, soit connue depuis longtemps [7], elle a pris un visage nouveau depuis une quinzaine d'années, avec la démonstration des multiples interactions entre le système immunitaire et des antigènes exogènes : tolérance fœtomaternelle [23], chimérisme [24], interactions avec des parasites [25], tolérance des micro-organismes commensaux [26], symbioses et même réponses immunitaires assurées par des bactéries [27]... Les bactéries commensales situées dans notre intestin, et sans lesquelles nous mourrions, ne sont-elles pas une part constitutive de nous-même? Les données récentes concernant la tolérance immunitaire montrent que les organismes sont en grande partie ouverts à leur extériorité et sont faits de cette extériorité.

Le modèle du soi a donc été remis en cause à la fois pour son imprécision conceptuelle et pour son inadéquation avec certaines données expérimentales. En même temps, cependant, que l'hypothèse du soi était critiquée, aucune autre théorie n'a réussi à s'imposer dans la discipline, si bien que celle-ci s'est trouvée en position de doute théorique. La réponse de la plupart des immunologistes a alors été d'accepter comme une bonne chose ce déficit de théorie [28]. Une telle position, néanmoins, est intenable: l'illusion qu'il pourrait y avoir des observations scientifiques sans théorie a été dissipée depuis longtemps. En effet, il n'y a de science que dans un cadre interprétatif fait de problèmes, de controverses et d'instruments, qui relèvent déjà de la théorie [29]. La véritable tâche des immunologistes est donc de proposer des modifications et améliorations aux théories existantes afin de dégager une nouvelle synthèse, qu'il conviendra évidemment de confronter aux données expérimentales, de manière à toujours viser l'hypothèse la plus adéquate possible.

\section{Comment renouveler le cadre théorique de l'immunologie? L’hétérogénéité contre l'internalisme}

Le modèle immunologique du soi et du non-soi est caractérisé par deux présupposés, l'autopréservation et une relative fermeture à l'extériorité. L'organisme est vu comme un être isolé et replié, c'est-à-dire fermé, indépendant et autonome [30]. Or, les observations récentes sur la tolérance suggèrent la fausseté de ce présupposé. II paraît donc nécessaire de promouvoir, contre l'internalisme, une compréhension de l'organisme comme réalité ouverte à son environnement, c'està-dire une réalité hétérogène en ce qu'elle accueille en son sein des éléments externes (parasites, bactéries...) et résulte de l'influence de ces éléments. Une vision non internaliste de l'organisme permet de comprendre celui-ci comme une réalité ouverte à l'extériorité, et construite dans et par cette extériorité. Comme le montre Jean-Claude Ameisen [31], le Même (l’organisme) est nécessairement, et dès sa formation, pénétré d'Autre (son environnement), et le système immunitaire a toujours affaire à du soi mêlé de non-soi. L'une des illustrations les plus frappantes de cette compénétration du soi et du non-soi est l'origine bactérienne des mitochondries chez les eucaryotes [32]: ces «usines énergétiques », indispensables au bon fonctionnement des cellules, dérivent de ce qui apparaît pourtant comme le cas le plus typique du non-soi dangereux, à savoir des bactéries.

Selon l'hypothèse de la continuité que nous avons proposée récemment [33], ce qui déclenche une réaction immunitaire effectrice n'est pas le non-soi, mais toute discontinuité forte dans les interactions entre les récepteurs des cellules immunitaires (récepteurs $B$, récepteurs $T$, récepteurs des macrophages...) et les motifs antigéniques auxquels ils réagissent, que ceux-ci soient exogènes (non-soi) ou endogènes (soi). Autrement dit, une réponse immunitaire effectrice se produit dès qu'apparaît entre les cellules immunitaires et leurs cibles un phénomène d'adhésion inhabituel (pour un compte rendu détaillé des conditions d'immunogénicité proposées par l'hypothèse de la continuité, voir [34]). Cela permet d'expliquer, par exemple, les réponses immunitaires déclenchées contre les cellules cancéreuses (les cellules cancéreuses sont bien des cellules du «soi » en tant qu'elles viennent du génome de l'organisme, et pourtant elles sont la cible d'une réponse immunitaire), ou encore la phagocytose, par les mêmes cellules immunitaires, de pathogènes aussi bien que de cellules mortes (qui sont censées faire partie du soi, mais qui expriment à leur surface des motifs antigéniques différents de ceux des cellules normales de l'organisme). Notre hypothèse propose aussi de penser certains phénomènes d'induction de tolérance comme des phénomènes d'induction de continuité : dans ce cas, un antigène nouveau introduit dans l'organisme en faibles quantités, à plusieurs reprises, sans dommage ni inflammation, induit une tolérance par continuité au lieu de provoquer une réponse immunitaire (désensibilisation pour les allergies, diminution des risques de rejet de greffe après introduction d'antigènes du donneur...). On peut interpréter de nombreux phénomènes de tolérance comme des phénomènes d'induction de continuité (tolérance fœtomaternelle, tolérance à certains parasites, chimérisme...), ce qui permet de rendre compte de l'ouverture relative de tout organisme aux entités étrangères. 


\section{Conclusions}

Le modèle du soi a été critiqué, au cours des trente dernières années, à la fois théoriquement et expérimentalement. Ces critiques ont conduit l'immunologie à une situation de doute théorique, marqué soit par une absence de théorie structurante, soit par l'idée erronée selon laquelle l'immunologie devrait se passer de théorie. II apparaît en réalité que, si l'on prolonge les thèses de l'immunité comme système autoréactif d'interactions par la fondation de l'idée d'une ouverture de l'organisme à l'extériorité par tolérance immunitaire, un renouvellement théorique est possible en immunologie. $\diamond$

\section{SUMMARY}

Uncertainties about the self and the issue of the proper theoretical model in immunology

Theoretical immunology constitutes a critical basis of all medical discoveries. Immunology has been dominated since the 1940 s by the self/nonself model. Here we try to shed light on the origins of this theoretical model and to show how and why this model has been called into question during the last thirty years. This paper has three aims. Firstly, we explore the sources of the immune self, going upstream from immunology to ecology-biology, psychology and eventually philosophy. Here the key questions: is the immune self really analogous with the philosophical and psychological selves in which it originates? What is the signification and adequacy of such a conceptual borrowing? We suggest that the «self» vocabulary in immunology is not clear and precise. Secondly, we present the experimental inadequacies of the self/nonself model. We show then how both the vagueness of the term «self» and these experimental flaws casted doubt on theories of immunology. Among the several models that have been proposed recently, none has attracted a consensus. Some immunologists have even suggested that immunology should rid itself of theorical concerns and concentrate on molecular aspects. This suggestion, however, is unacceptable; hence it is still necessary to find a theoretical framework for immunology. Finally, we try to suggest a way to escape this uncomfortable situation of doubt. The immune «self» and the immune «system » («network») are rooted in strong metaphysical conceptions of identity, the main characteristic of which is to consider the organism as an enclosed and self-constructing entity. By contrast, based on experimental data about immune tolerance and host-pathogen interactions, we propose to consider organisms as open entities. To what theory does this conception lead? What would be the consequences of such a theory with regard to medical aspects? $\diamond$

\section{RéFÉRENCES}

1. Moulin AM. Le dernier langage de la médecine. Histoire de l'immunologie de Pasteur au Sida. Paris: PUF, $1991: 448 \mathrm{p}$.

2. Burnet FM. The Darwinian approach to immunity. In : Sterzl J, ed. Molecular and cellular basis of antibody formation. New York: Academic Press, 1965 : 17-20.

3. Tauber Al. The immune self. Theory or metaphor? Cambridge : Cambridge University Press, $1994: 354 \mathrm{p}$.

4. Metchnikoff $\varepsilon$. L'immunité dans les maladies infectieuses. Paris : Masson, 1901: 600 p.
5. Burnet FM, Fenner F. The production of antibodies, $2^{\text {nd }}$ ed. Melbourne : MacMillan, 1941, 1949: 142 p.

6. Burnet FM. Changing patterns. An atypical autobiography. Melbourne: Heinemann, $1968: 282 \mathrm{p}$.

7. Billingham RE, Brent L, Medawar PB. Actively acquired tolerance of foreign cells. Nature $1953 ; 172: 603-6$.

8. Kappler JW, Roehm N, Marrack, PT. T cell tolerance by clonal elimination in the thymus. Cell $1987 ; 49: 273-80$.

9. Dausset J. La définition biologique du soi. In : Bernard J, Bessis M, Debru C, eds. Soi et non-soi. Paris : Seuil, 1990 : 19-26.

10. Ehrlich P. On immunity with special reference to cell life. Proc Roy Soc $1900 ; 66: 424-48$.

11. Ehrlich P, Morgenroth J. Über Hämolysine : fünfte Mittheilung. Berliner Klinische Wochenschrift 1901; 38:251-6.

12. Moulin AM. La métaphore du soi et le tabou de l'auto-immunité. In : Bernard J, Bessis M, Debru C, eds. Soi et non-soi. Paris : Seuil, 1990 : 55 68.

13. Wells HG, Huxley JS, Wells GP. The science of life. New York: The Literary Guild, 1929, 1934 : 1514 p.

14. Burnet FM. Biological aspects of infectious disease. New York: MacMillan, $1940: 310 \mathrm{p}$.

15. Burnet FM, Fenner F. Genetics and immunology. Heredity $1948 ; 2$ : 289 324.

16. Locke J. Essai sur l'entendement humain (1690). Traduction J.M. Vienne. Livres I et II. Paris: Vrin, $2001: 640$ p.

17. Löwy I. The immunological construction of the self. In : Tauber Al, ed. Organism and the origins of self. Boston : Kluwer, 1991: 43-75.

18. Burnet FM. Immunological surveillance. Sydney: Pergamon, $1970: 280$ p.

19. Silverstein AM, Rose NR. There is only one immune system! The view from immunopathology. Sem Immunol 2000 ; 12 : 173-8.

20. Hempel C. Éléments d'épistémologie. Paris : Armand Colin, 1972: 184 p.

21. Freitas AA, Rocha B. Peripheral T cell survival. Curr Opin Immunol 1999; $11: 152-6$.

22. Chatenoud L, Salomon B, Bluestone JA. Suppressor T cells. They're back and critical for regulation of autoimmunity! Immunol Rev 2001; 182 : 149-63.

23. Carosella ED, Moreau P, Le Maoult J, et al. HLA-G molecules: from maternal-fetal tolerance to tissue acceptance. Adv Immunol 2003 ; 81 : 199-252.

24. Bianchi DW, Zickwolf GK, Weil GJ, et al. Male fetal progenitor cells persist in maternal blood for as long as 27 years postpartum. Proc Natl Acad Sci USA $1996 ; 93: 705-7$.

25. Combes C. Interactions durables. Écologie et évolution du parasitisme. Paris : Masson, $524 \mathrm{p}$.

26. Hooper LV, Gordon JI. Commensal host-bacterial relationships in the gut. Science $2001 ; 292$ : 1115-8.

27. Noverr MC, Huffnagle GB. Does the microbiota regulate immune responses outside the gut? Trends Microbiol $2004 ; 12$ : 562-8.

28. Vance RE. A Copernician revolution? Doubts about the danger theory. J Immunol $2000 ; 165$ : 1725-8.

29. Bachelard G. Le nouvel esprit scientifique (1934). Paris: PUF, 1995 : $186 \mathrm{p}$.

30. Wilson D. The science of self. A report of the new immunology. London: Longman, 1972 : $346 \mathrm{p}$.

31. Ameisen JC. La sculpture du vivant. Le suicide cellulaire ou la mort créatrice. Paris : Seuil, 2003, 458 p.

32. Margulis L, Chapman MJ. Endosymbioses: cyclical and permanent in evolution. Trends Microbiol 1998 ; 6 : 342-5.

33. Pradeu T, Carosella $\varepsilon D$. Analyse critique du modèle immunologique du soi et du non-soi et de ses fondements métaphysiques implicites. CR Acad Sci Paris Biologie 2004 ; 327 : 481-92.

34. Pradeu T, Carosella ED. The self model and the conception of biological identity in immunology. Biology and Philosophy 2005 (sous presse).

\section{REMERCIEMENTS}

À Anouk Barberousse, Cédric Brun, Edgardo D. Carosella, Jean Gayon, Anne-Marie Moulin, Alfred Tauber et Guy-Cédric Werlings.

\section{TIRÉS À PART}

\section{T. Pradeu}

